\title{
Surgical Technique of Vertebral Body Removal and Anterior Reconstruction in L5 Spondylectomy
}

\author{
Apiruk Sangsin, Hideki Murakami, Takaki Shimizu, Satoshi Kato, Satoru Demura and Hiroyuki Tsuchiya \\ Department of Orthopaedic Surgery, Graduate School of Medical Sciences, Kanazawa University, Kanazawa, Japan
}

\begin{abstract}
:
Introduction: L5 spondylectomy for the treatment of spinal tumor is a technically demanding surgery because of the complex anatomy of major vessels, the obscurity of the posterior exposure from the iliac wings, and the increased comparative size of the L5 vertebral body. In this study, we present a surgical technique of L5 spondylectomy, vertebral body removal, and anterior reconstruction for a case with solitary spinal metastatic renal cell carcinoma (RCC).

Technical Note: A 54-year-old man underwent right total nephrectomy for RCC one year ago. At the one-year postoperative follow-up, CT scan and MRI revealed a solitary L5 spinal metastasis. A two-stage posteroanterior approach was performed. To facilitate vertebral body removal, transverse processes were separated from the vertebral body by using the posterior approach. On the basis of the anterior approach, the vertebral body was removed via the interval space between the left common iliac vessels. Reconstruction was performed by using a liquid-nitrogen-frozen, tumor-bearing bone mixed with an autogenous bone graft in an expandable titanium cage.

Results: No intraoperative complications were observed. Postoperatively, the patient exhibited muscle weakness in the tibialis anterior and extensor hallucis longus bilaterally but improved with time. Seven months after the operation, the patient was able to walk independently. At the recent 2.5-year follow-up, the local recurrence of lesions was nonexistent. The bone graft had fused with the adjacent vertebrae.

Conclusion: This report described a novel technique for L5 spondylectomy that can facilitate safe L5 vertebral body removal and demonstrated the effectiveness of liquid-nitrogen-frozen, tumor-bearing bone mixed with autogenous bone graft in anterior reconstruction both in terms of oncologic safety and biological healing.
\end{abstract}

Keywords:

Renal cell carcinoma, spinal metastasis, spondylectomy, spinal tumor

Spine Surg Relat Res 2018; 2(3): 236-242 dx.doi.org/10.22603/ssrr.2018-0014

\section{Introduction}

One-third of metastatic renal cell carcinoma (mRCC) patients experience osseous metastasis ${ }^{1}$, most of which involve the spine ${ }^{2}$. Owing to improvements in surgical techniques and preoperative embolization, spondylectomy in spinal mRCC is now considered safe and effective for local con$\mathrm{trol}^{3)}$. Moreover, it can prolong the survival of spinal mRCC patients with solitary lesions ${ }^{4}$. Unlike spondylectomy in the thoracic spine, L5 spondylectomy for the treatment of spinal tumors is a technically demanding surgery because of the unique anatomy of the spine. Achieving a wide or marginal margin of a spinal tumor is the goal. Unfortunately, this procedure is associated with a risk of major neurovascular inju- ries, particularly during anterior vertebral body removal, where it overlays the bifurcation of the common iliac veins. In this report, we present a surgical technique for L5 spondylectomy, vertebral body removal, and anterior reconstruction in the case of a patient with spinal $\mathrm{mRCC}$.

\section{Patients and Methods}

A 54-year-old man underwent right total nephrectomy for RCC one year ago. At the one-year postoperative follow-up, computed tomography (CT) scan and magnetic resonance imaging (MRI) revealed a solitary L5 spinal metastasis (Fig. 1). Two months after the diagnosis of spinal metastasis, the following were performed: a two-stage posteroanterior ap-

Corresponding author: Hideki Murakami, hmuraka@med.kanazawa-u.ac.jp

Received: April 2, 2018, Accepted: May 4, 2018

Copyright (C) 2018 The Japanese Society for Spine Surgery and Related Research 


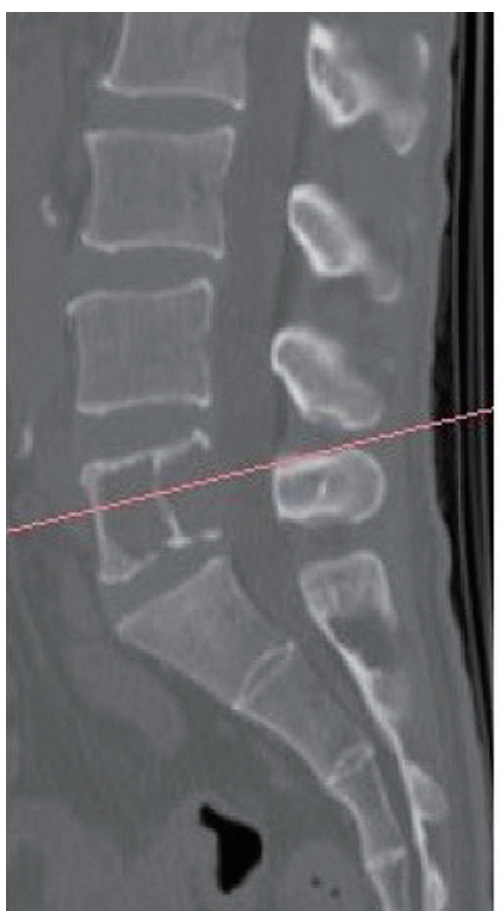

A
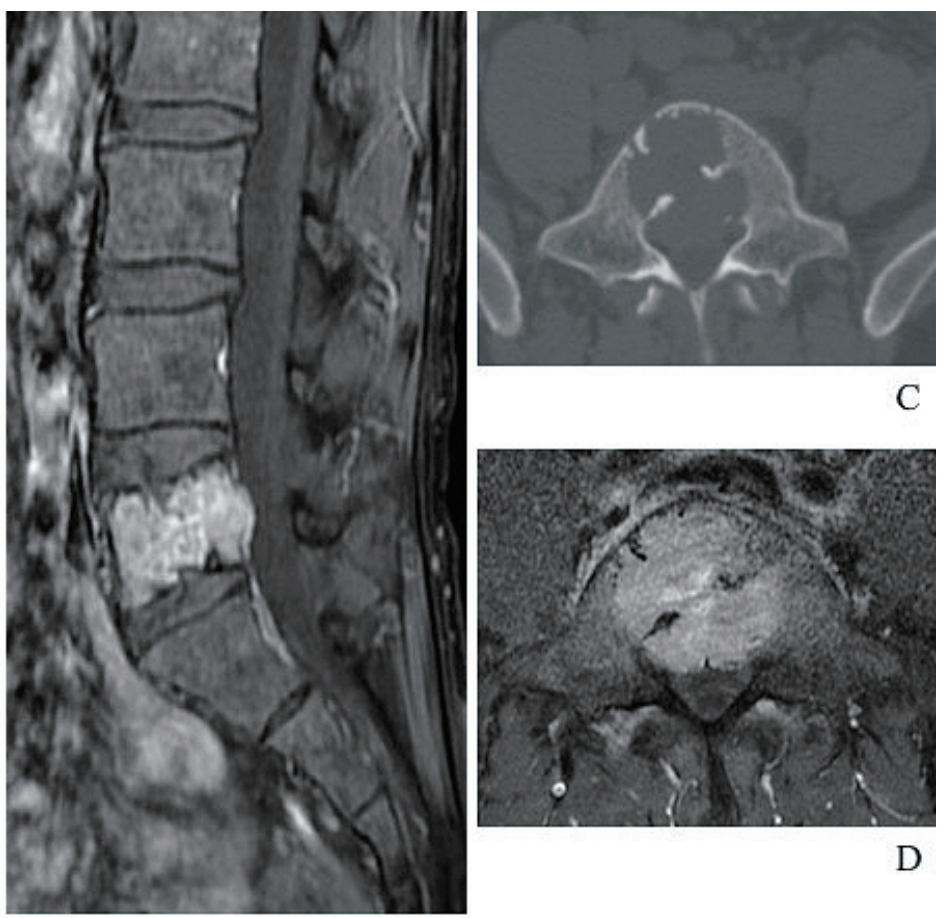

B

Figure 1. CT (A) and sagittal MRI (B) demonstrating the tumor involvement of the L5 vertebral body, as well as intact pedicles and transverse processes bilaterally. Axial CT (C) and MRI (B) demonstrating tumor extension into the spinal canal.

proach, L5 spondylectomy, and reconstruction with a liquidnitrogen-frozen, tumor-bearing bone mixed with an autogenous bone graft in an expandable titanium cage.

\section{Technical Note}

\section{Step 1: Preoperative embolization}

Right L4 segmental, median sacral, and bilateral iliolumbar arteries were preoperatively embolized two days before the surgery.

\section{Step 2: Posterior Approach}

The patient was placed in a prone position. A straight midline incision extending from the L3 to the tip of the sacrum was made. The paraspinal muscles were dissected and then retracted laterally away from the spinous processes and laminae. The inferior articular processes and spinous process of L4 were removed to expose the superior articular processes of L5. The soft tissue attached to the inferior portion of the pars interarticularis was dissected with care to avoid L5 root damage. A T-saw guide was inserted through the intervertebral foramen in the cephalad to caudad direction. A flexible multifilament thread wire (T-saw; Pro Medical, Kanazawa, Japan) was passed through the T-saw guide. The Tsaw was wrapped around the pedicle and clamped with the T-saw holder at both ends, and the pedicles were cut using the reciprocating motion of the T-saw. By this technique, the posterior elements of L5 including the lamina, spinous proc- ess, and superior and inferior articular processes were removed in one piece (Fig. 2). The cut surface of the pedicles was sealed with bone wax for hemostasis and to minimize tumor cell contamination. Thereafter, the dural tube was dissected from the posterior longitudinal ligament and the dural attachment complex. The L5-adjacent discs were removed using a pituitary rongeur and a curette. Cutting lines were made between the pedicles and the transverse processes bilaterally by using a high-speed drill for ease of anterior vertebral body removal. Care was taken not to violate the tumor margin (Fig. 3). A Gore-Tex Patch (WL Gore \& Associates, Inc., Flagstaff, AZ) composed of artificial soft tissue was laid between the dural sac and the L5 vertebral body as a landmark for the anterior approach. Bilateral L3, L4, and S1 pedicle and sacroiliac screws were inserted and affixed to the rods (Fig. 4). Finally, the right posterior iliac crest bone graft was harvested for anterior reconstruction.

\section{Step 3: Anterior Approach}

An anterior, midline, transperitoneal approach was performed. The common iliac arteries and veins were dissected away from the L5 vertebral body and the adjacent intervertebral discs (Fig. 5). The interval space between the left common iliac artery and vein was assessed to perform the $\mathrm{L}$ 5 vertebral body removal (Fig. 6). The bilateral transverse processes were left in place. The excised tumor-bearing bone was immersed in liquid nitrogen at $-196^{\circ} \mathrm{C}$ for $20 \mathrm{~min}$, cut into small pieces, mixed with the autogenous bone graft, and packed into a titanium expandable cage. The cage was 


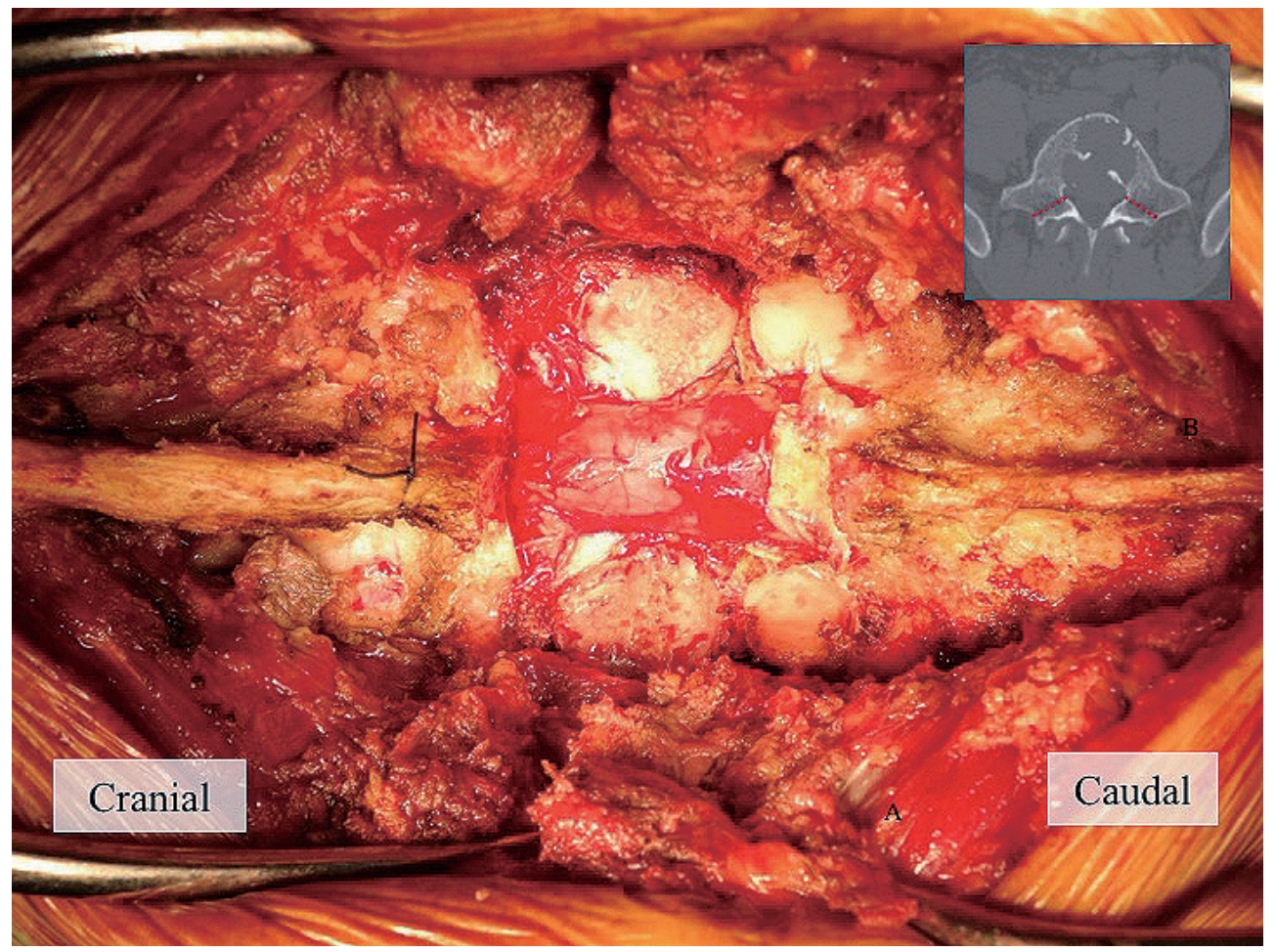

Figure 2. The lamina, spinous process, and superior and inferior articular processes were removed in one piece by using a T-saw. The cutting lines were illustrated as red dotted lines on the axial CT imaging of L5 (right upper corner).

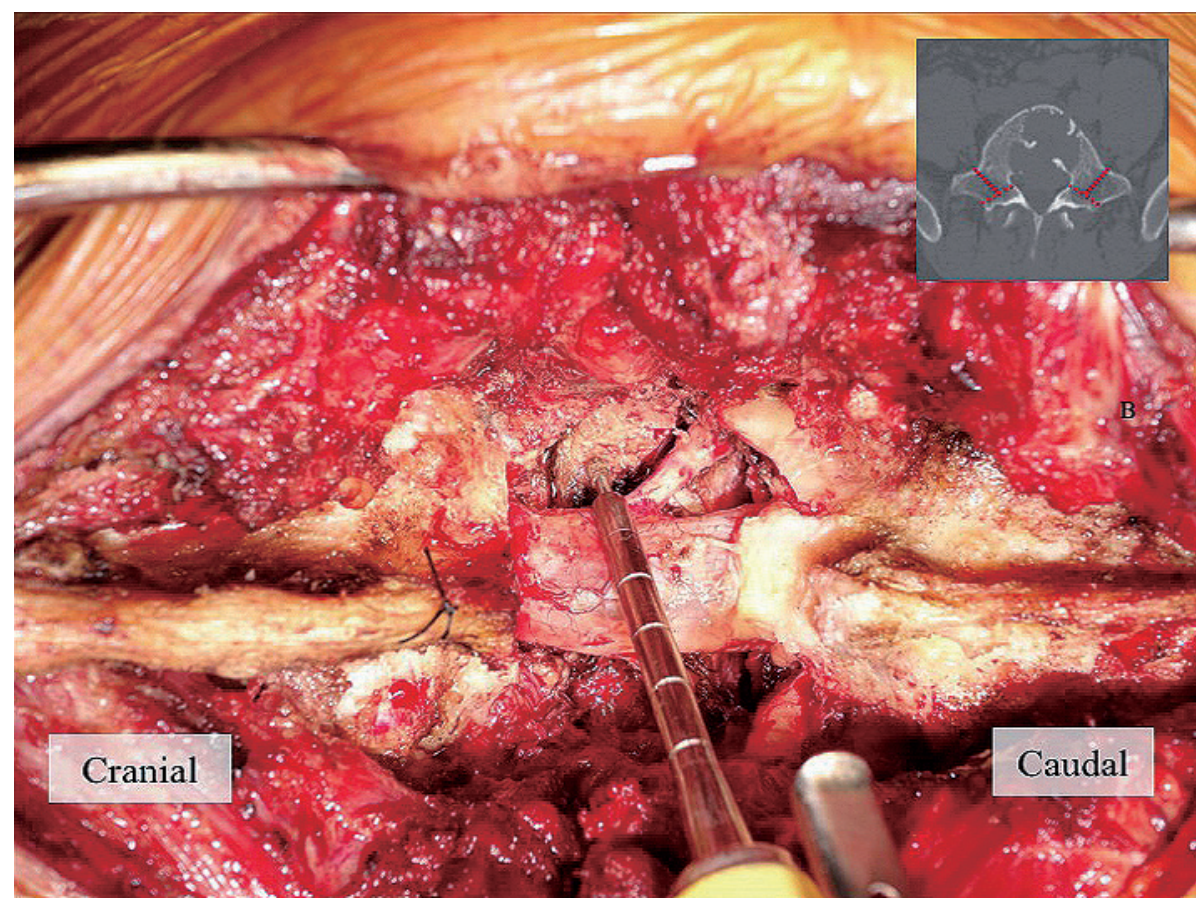

Figure 3. Second cutting lines were made between the junction of the transverse processes and pedicles bilaterally to separate the transverse processes from the vertebral body (indicated by the red lines on the axial $\mathrm{CT}$ at the right upper corner). 


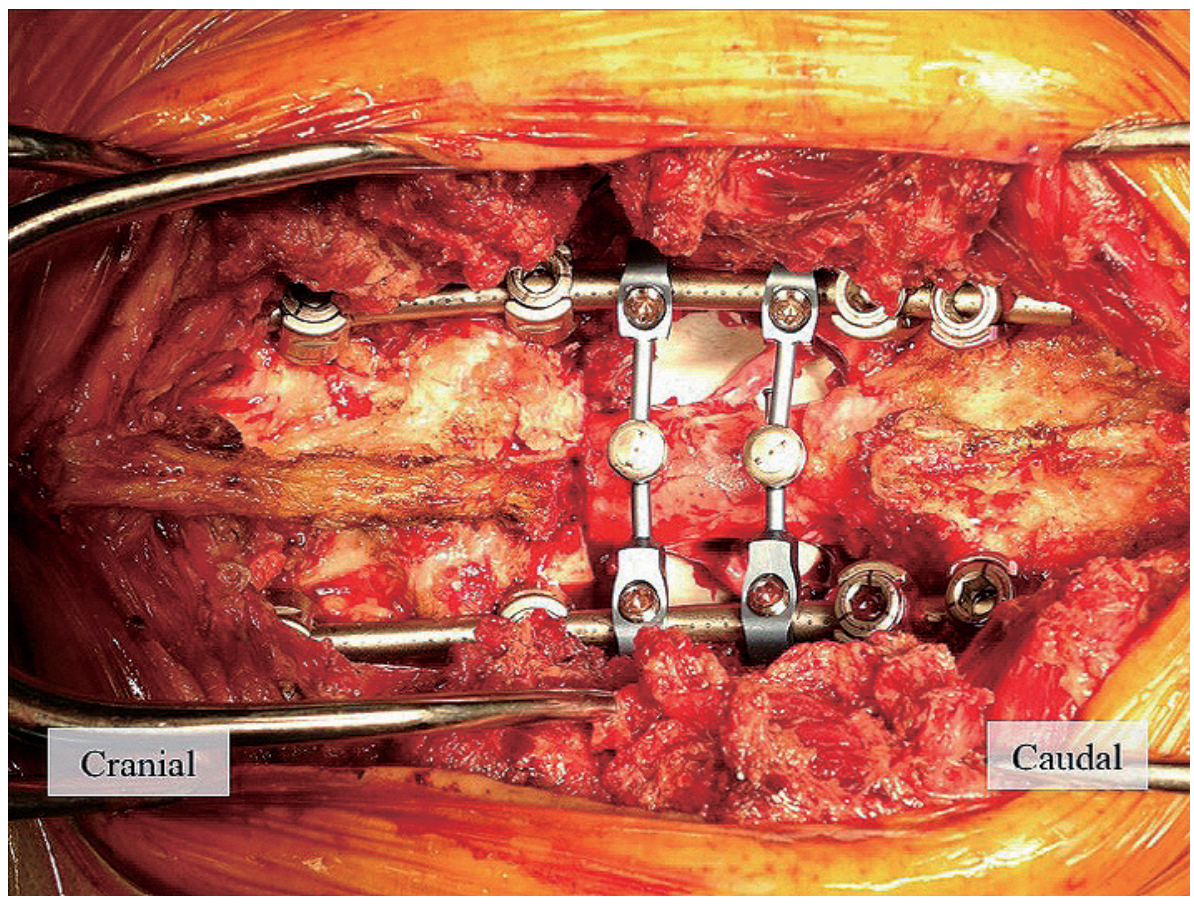

Figure 4. An intraoperative photograph demonstrating posterior instrumentation using bilateral L3, L4, and S1 pedicle and sacroiliac screws. The artificial sheet was laid between the dural sac and the L5 vertebral body as a landmark for an anterior approach.

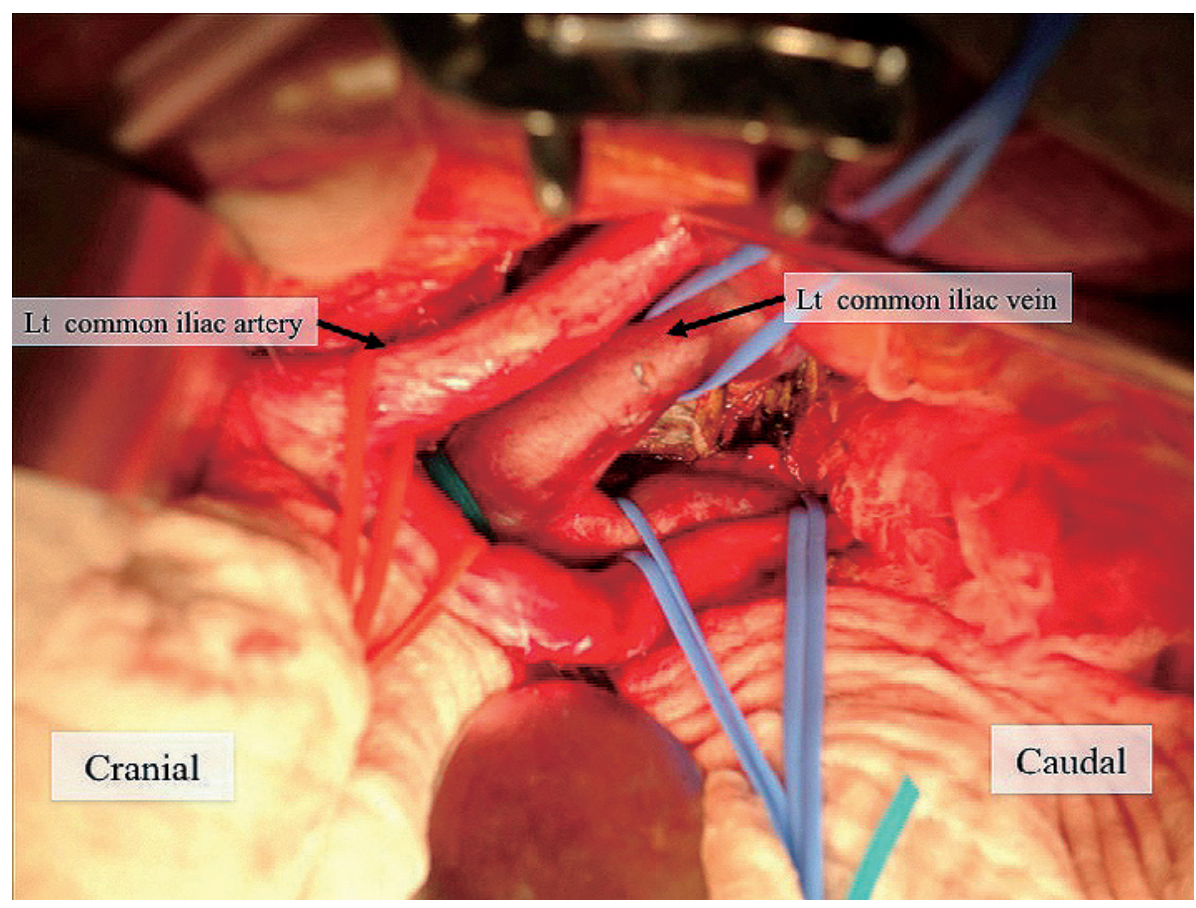

Figure 5. Bilateral iliac vessels were dissected free from the anterior aspect of the L5 vertebral body and adjacent discs.

then used to replace the removed vertebral body.

\section{Results}

\section{Pathological findings}

The pathological findings of the affected vertebrae were consistent with a diagnosis of mRCC.

\section{Postoperative course}

Postoperatively, the patient exhibited muscle weakness in the right and left tibialis anterior (TA) muscles (grades 3 and 1) and the extensor hallucis longus (EHL) (grades 3 and 1). Inpatient rehabilitation was started two days after the 


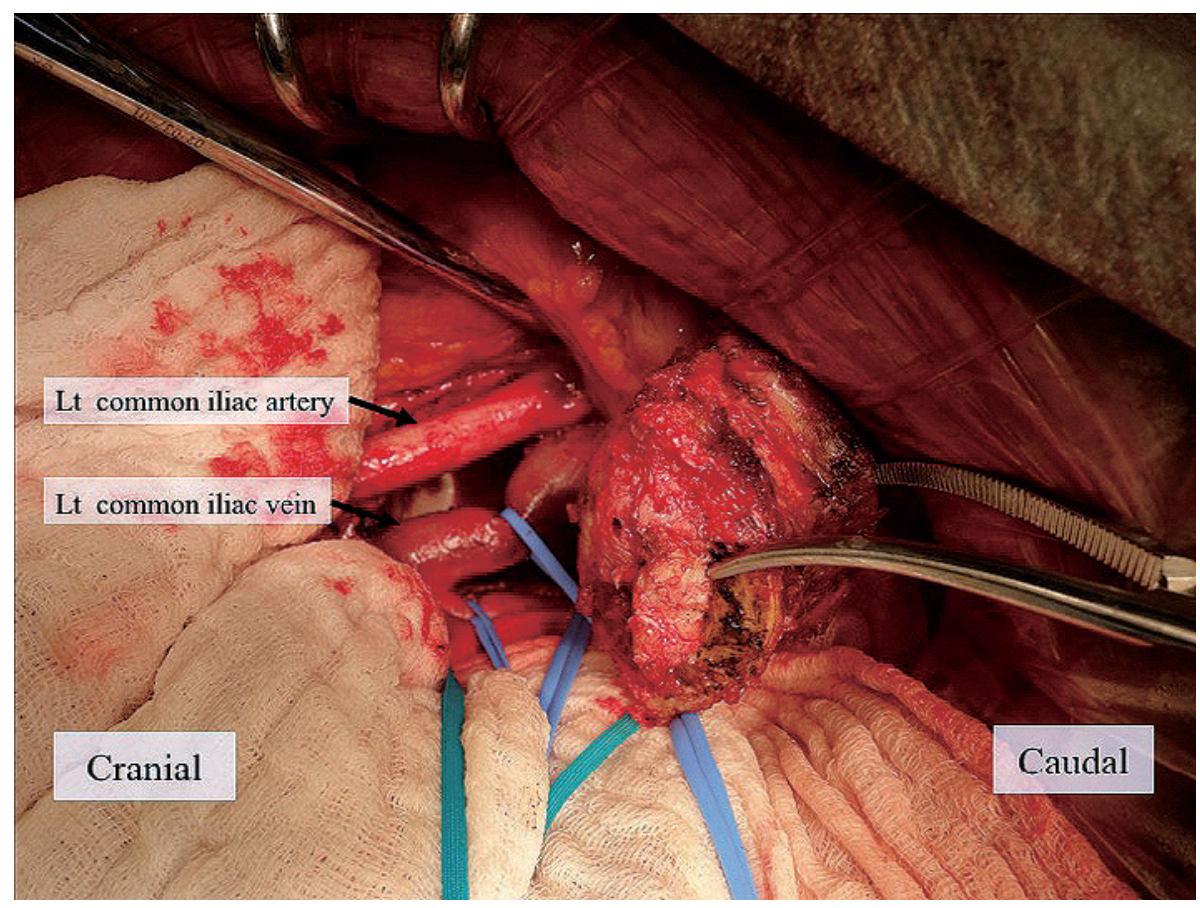

Figure 6. The L5 vertebral body was removed between the interval of the left common iliac vein and artery.
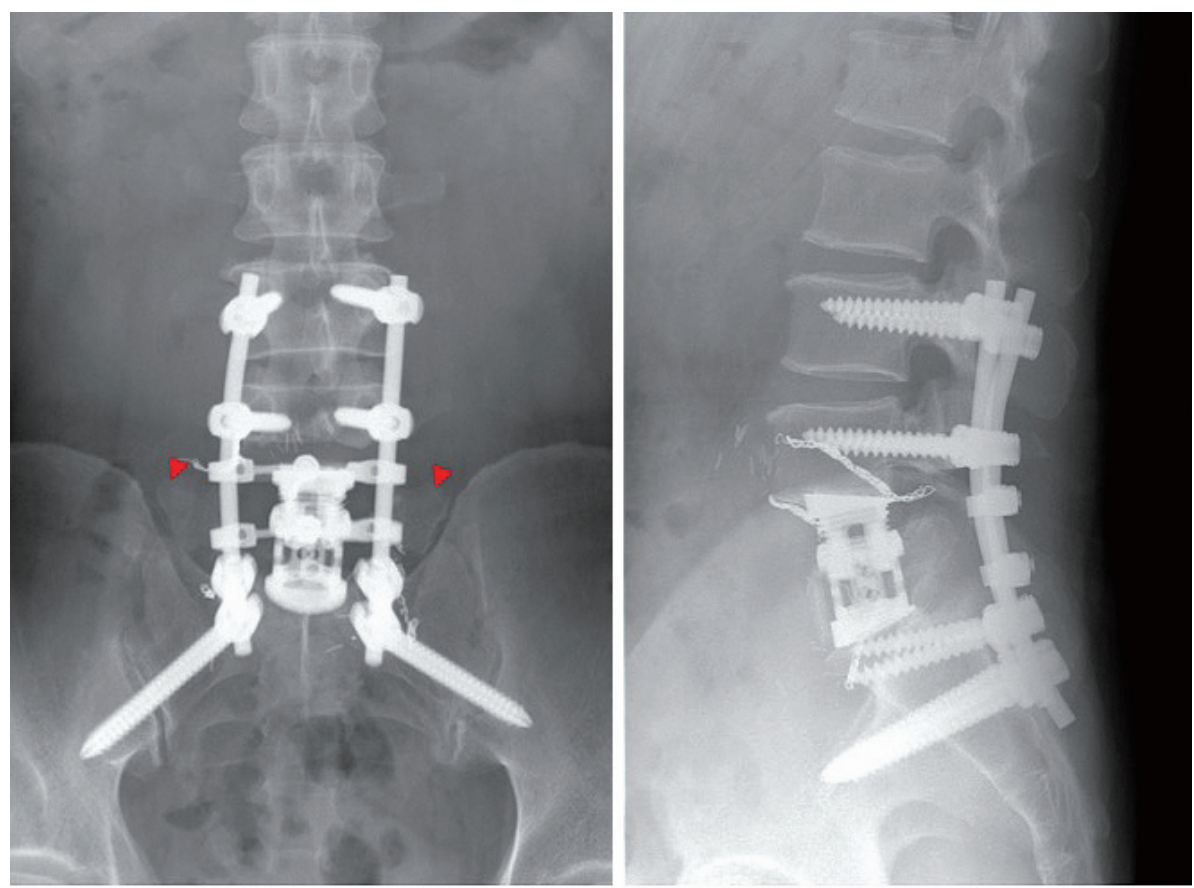

A

Figure 7. A recent 2.5-year follow-up demonstrating well-maintained instrumentation and spinal alignment. Transverse processes were left in place (red arrow heads).

surgery. The patient was discharged home on postoperative day 38 after the complete rehabilitation program. By the time of discharge, the patient could walk steadily with a cane. Seven months after the operation, the muscle power of his right and left TA improved to grades 4 and 2, respectively, and he could walk independently. Unfortunately, at that time, the muscle power of the EHL had not improved.
At the one-year follow-up after the surgery, radiographic union had been achieved between the bone graft site and the adjacent vertebrae. At the recent 2.5-year follow-up, postoperative radiograph (Fig. 7) and CT scan (Fig. 8) showed well-maintained instrumentation, with a radiographic evidence of a bony union between the adjacent vertebrae and the bone graft. No evidence of local recurrence at the L5 

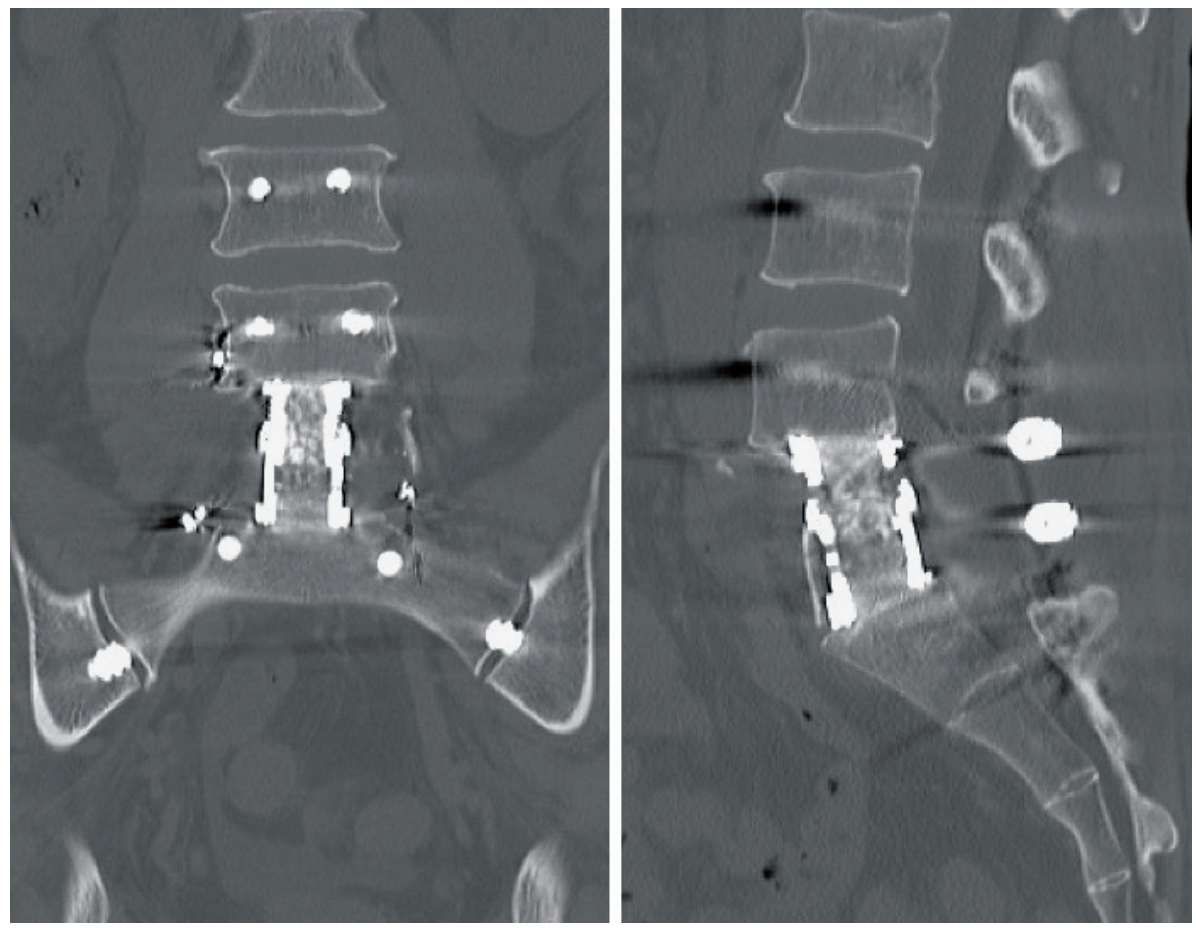

Figure 8. A recent 2.5-year follow-up CT scan demonstrating a solid bony union between the bone graft site and adjacent vertebrae. No local recurrence was observed.

spondylectomy site was observed.

\section{Discussion}

Unlike the spondylectomy of the thoracic vertebrae or L1, which requires only a one-stage posterior approach operation, L5 spondylectomy requires a two-stage posteroanterior approach operation because of the complex anatomy of the major vessels, obscurity of the posterior exposure from the iliac wings, and the increased comparative size of the L5 vertebral body, which may injure the lumbar nerve roots during rolling out posteriorly. At the first stage posterior operation, Gallia et al. ${ }^{5}$ reported the case of an L5 spondylectomy wherein all of the L5 posterior elements, including the lamina, spinous process, superior and inferior articular processes, and transverse processes, were removed en bloc; however, the technique was not mentioned in detail. On the basis of our technique, we found that during the posterior approach operative stage, the removal of the transverse processes in one piece and other posterior elements was impossible because of the blockage by the iliac wings. Thus, transverse processes must be removed together with the vertebral body during the second stage anterior operation.

In the past, during the anterior approach, the vertebral body that attaches to the transverse processes through the interval space between the left common iliac artery and vein was removed after the dissection of the L5 vertebral body from the surrounding structures and adjacent discs. This approach required sacrificing the left common iliac vein because the transverse processes could get stuck to the left common iliac vessels, thus leading to uncontrollable vascu- lar injury. Even though there have been no reported complications after left iliac vein ligation, left iliac vein occlusion can lead to an unpredictable spectrum of symptoms ranging from no symptoms to severe phlegmasia cerulea dolens ${ }^{6}$. Therefore, we tried to find the best way to preserve the left common iliac vein during the anterior L5 vertebral body removal in cases wherein the L5 transverse processes are not involved in the tumor.

In this case, the tumor did not involve the pedicles and transverse processes; thus, the transverse processes were kept in their place to facilitate the ease of anterior vertebral body removal. After cutting and removing the lamina and spinous process posteriorly, second cutting lines were made between the junction of the pedicles and transverse processes bilaterally (Fig. 3). Thereafter, the vertebral body could be easily removed via the interval space between the left common iliac vein and artery during the anterior operation (Fig. 6). Owing to the rarity of these cases, the safe interval space for the L5 vertebral body removal between the common iliac vessels still has no consensus. Some surgeons have removed the L5 vertebral body via the interval space between the bifurcation of the common iliac vessels ${ }^{5}$. However, the cranial retraction of the common iliac vessels may cause vascular injury during L5 vertebral body extraction ${ }^{7}$, particularly to the fragile common iliac veins because the bifurcation of the common iliac veins is located anterior to the L5 (Fig. 5) but not the L4 vertebral body, as illustrated in a report ${ }^{5}$. Therefore, from our experience, we determined that safe vertebral body removal was impossible via the interval space between the bilateral common iliac veins.

The anterior vertebral reconstruction of an L5 vertebrec- 
tomy has many options. We suggest using an expandable lordotic cage filled with liquid-nitrogen-frozen, tumorbearing bone mixed with autogenous bone grafts. The exact height can be adjusted to achieve primary stability such that a third stage posterior approach ${ }^{8)}$ for compression by posterior instrumentation is unnecessary. By using liquidnitrogen-frozen, tumor-bearing bone grafts, we can reduce the operative time, blood loss, and autograft site morbidity and create a systemic antitumor immune response ${ }^{9-12)}$.

In this report, we described a technique for L5 spondylectomy in which the tumor affected only the vertebral body by creating cutting lines between the pedicles and transverse processes bilaterally for increased safety during anterior L5 vertebral body removal. We have also suggested that anterior L5 vertebral body removal should be conducted via the interval space between the left common iliac vessels. For anterior reconstruction, the use of an expandable lordotic cage filled with liquid-nitrogen-frozen, tumor-bearing bone mixed with autogenous bone graft is an effective option for anterior reconstruction both in terms of oncologic safety and biological healing.

Conflicts of Interest: The authors declare that there are no conflicts of interest.

Acknowledgement: We would like to thank Editage (www.editage.com) for the English language editing.

Author Contributions: AS wrote and prepared the manuscript, HM, TS, SK and SD performed the surgery and reviewed the manuscript, and HM and HT approved the manuscript. All authors have read, reviewed, and approved the manuscript.

\section{References}

1. Bianchi M, Sun M, Jeldres C, et al. Distribution of metastatic sites in renal cell carcinoma: a population-based analysis. Ann Oncol. 2012;23(4):973-80.

2. Rose PS, Buchowski JM. Metastatic disease in the thoracic and lumbar spine: evaluation and management. J Am Acad Orthop Surg. 2011;19(1):37-48.

3. Kawahara N, Tomita K, Murakami H, et al. Total en bloc spondylectomy for spinal tumors: surgical techniques and related basic background. Orthop Clin North Am. 2009;40(1):47-63, vi.

4. Kato S, Murakami H, Demura S, et al. Spinal metastasectomy of renal cell carcinoma: a 16-year single center experience with a minimum 3-year follow-up. J Surg Oncol. 2016;113(5):587-92.

5. Gallia GL, Sciubba DM, Bydon A, et al. Total L-5 spondylectomy and reconstruction of the lumbosacral junction. Technical note. J Neurosurg Spine. 2007;7(1):103-11.

6. Murray JD, Brennan FJ, Hall LD, et al. Left iliac vein occlusion: its clinical spectrum. Ann Vasc Surg. 2000;14(5):510-6.

7. Wenger M, Teuscher J, Markwalder R, et al. Total spondylectomy and circular reconstruction for L5 vertebral body chordoma using a telescopic lordotic cage. Acta Orthop. 2006;77(5):825-9.

8. Kawahara N, Tomita K, Murakami H, et al. Total en bloc spondylectomy of the lower lumbar spine: a surgical techniques of combined posterior-anterior approach. Spine (Phila Pa 1976). 2011;36(1):74-82.

9. Murakami H, Kato S, Demura S, et al. Novel reconstruction technique using a frozen tumor-bearing vertebra from a total en bloc sponydylectomy for spinal tumors. Orthopedics. 2013;36(8):605-7.

10. Chu KF, Dupuy DE. Thermal ablation of tumours: biological mechanisms and advances in therapy. Nat Rev Cancer. 2014;14(3): 199-208.

11. Murakami H, Demura S, Kato S, et al. Increase of IL-12 following reconstruction for total en bloc spondylectomy using frozen autografts treated with liquid nitrogen. PLoS One. 2013;8(5):e 64818.

12. Murakami H, Demura $S$, Kato $S$, et al. Systemic antitumor immune response following reconstruction using frozen autografts for total en bloc spondylectomy. Spine J. 2014;14(8):1567-71.

Spine Surgery and Related Research is an Open Access article distributed under the Creative Commons Attribution-NonCommercial-NoDerivatives 4.0 International License. To view the details of this license, please visit (https://creativeco mmons.org/licenses/by-nc-nd/4.0/). 